\title{
Kunstvolles Recycling von alten Röntgenkästen
}

\section{Daniel Lüthi}

Freier Journalist und Fotograf, Medientrainer, Bern

In den Arztpraxen zeigen sie schwarz-weiss die oft brutale medizinische Realität: die Leuchtkästen, die Röntgenbilder sichtbar machen. Die Berner Künstlerin Judith Zaugg sorgt dafür, dass die Kästen nach ihrer Ausmusterung in veränderter Form eine neue Bedeutung erlangen. Als dekorative Elemente, die mit einer farbigen und fröhlichen Fantasiewelt Praxen - und die Augen von Patientinnen zum Leuchten bringen.

Es war eine Gynäkologin, die Judith Zaugg auf die Idee brachte: "An einer Ausstellung von mir sah sie vor etwa zehn Jahren Leuchtkästen, die ich in Brockenhäusern und im Abfall zusammengesammelt hatte, zum Beispiel Geleisebeschriftungen der SBB oder Hinweise der Post. Als Ärztin dachte sie sofort an
Röntgenkästen, die sich für meine Art von Recycling anbieten würden.» Es stellte sich heraus, dass sie sich für diesen Zweck besonders gut eignen, weil sie ein gleichmässiges Licht abgeben. Die Gynäkologin gab Zaugg auch gerade einen Auftrag: Sie bestellte zwei Bilder, die in die Praxis einer Frauenärztin passen.

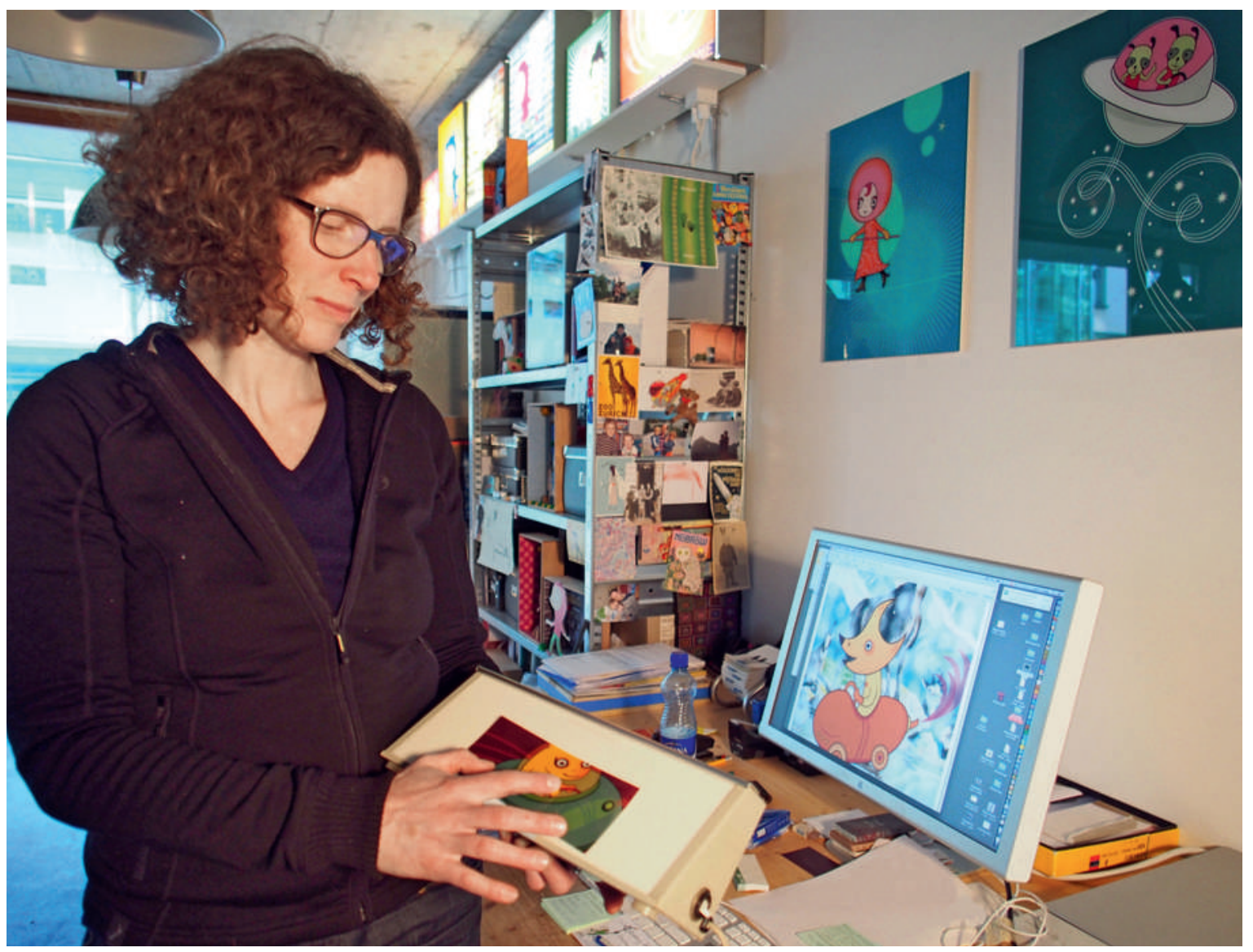

Judith Zaugg in ihrem Atelier: Ein kleiner Röntgenkasten erhält eine neue Funktion. 


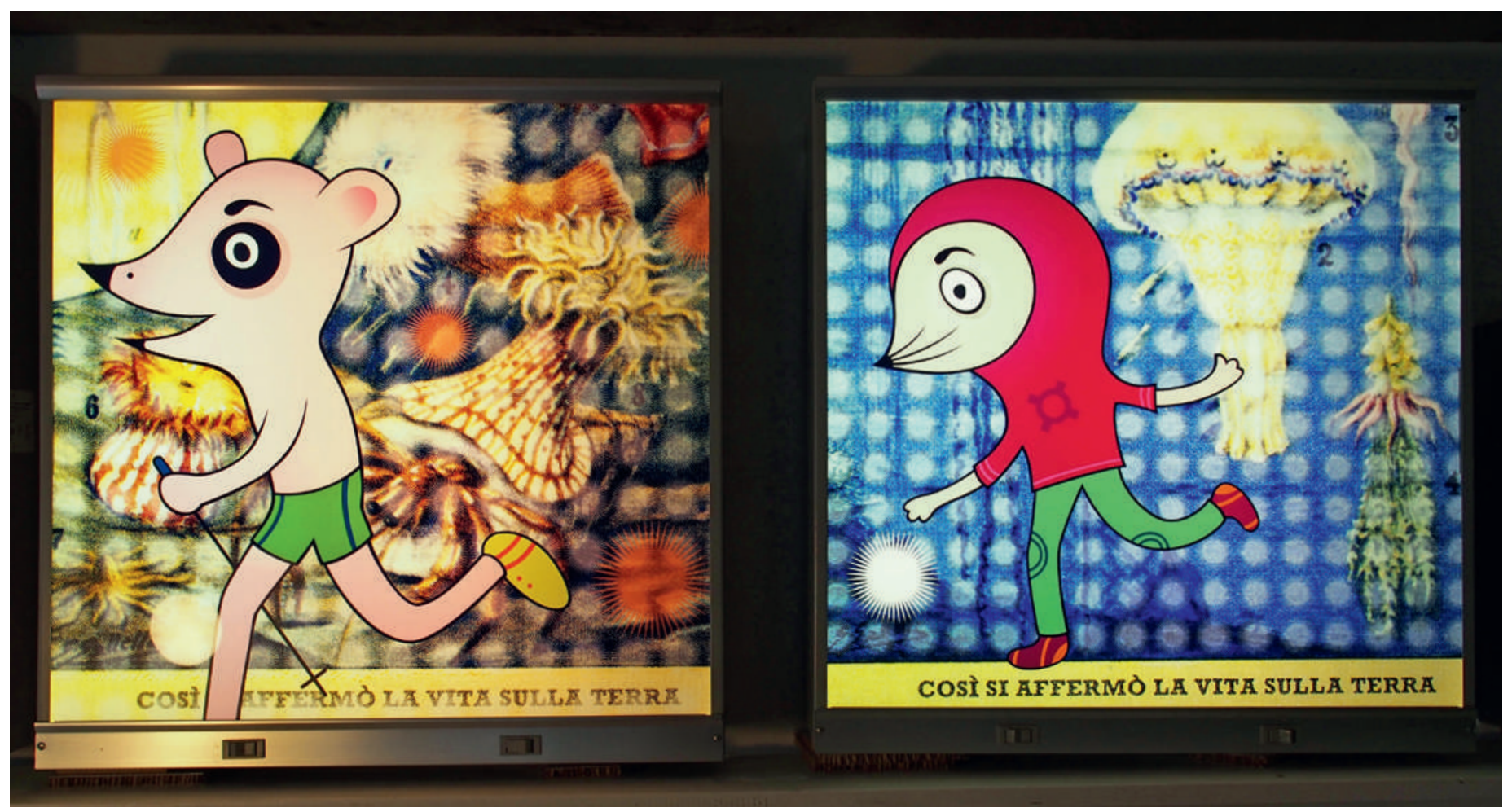

Aus Schwarzweiss-Betrachtern sind leuchtende Fantasiewelten geworden.

\section{Zeichnen und konstruieren}

Der kreative Prozess beginnt ganz traditionell mit einer Bleistift-Skizze. Bald aber wechselt Judith Zaugg an den Computer, wo sie ihre Idee im IllustratorProgramm weiterentwickelt: «Zeichnen bedeutet hier (grafisch konstruieren». Mithilfe von Vektoren lasse ich einzelne Flächen in verschiedenen Formen entstehen. Diese kann ich dann beliebig platzieren,

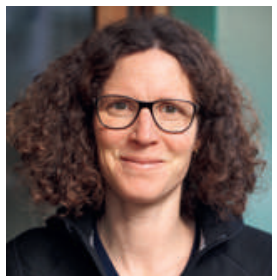

\section{Judith Zaugg}

Grafikerin, Künstlerin,

Leuchtkasten-Spezialistin

Judith Zaugg wurde 1970 in Bern geboren. An der Berner Schule für Gestaltung besuchte sie den Vorkurs und die Fachklasse für Grafik. 1993 bis 1998 war sie Mitglied der ComicGruppe "Gangloff». Seit 1997 arbeitet sie als selbständige Grafikerin, Illustratorin und Künstlerin. Eine ihrer Spezialitäten ist es, aus alten Röntgenkästen leuchtende Kunstwerke zu machen. Judith Zaugg hat verschiedene Preise und Stipendien erhalten und im In- und Ausland zahlreiche Ausstellungen gemacht. Sie ist verheiratet, Mutter von drei Söhnen und lebt in Bern. grösser oder kleiner machen, verschieden einfärben, verformen und verschieben. Es ist eine Art grafisches Spiel, das ich sehr gerne habe.» Wenn das Bild fertig ist, wird es, wie eine Fotografie, auf eine Folie belichtet. Diese kommt zwischen zwei Plexiglasscheiben, und das Ganze ersetzt dann die bisherige Abdeckung des Röntgenkastens.

Die ersten Leuchtkästen der Berner Grafikerin und Künstlerin entstanden etwa im Jahr 200o. «Beim Zeichnen meiner digitalen Bilder war ich immer wieder fasziniert von den leuchtenden Farben meiner Illustrationen am Bildschirm. Ich suchte nach Möglichkeiten, ihnen diese Leuchtkraft zu erhalten. Auf einer Reise durch das Hinterland Kataloniens entdeckte ich dann vielerorts alte Werbeleuchtkästen. Diese brachten mich auf die Idee, fiktive Werbungen zu erfinden und auf alten Kästen leuchten zu lassen.»

Die Kästen sind zu einer Spezialität, ja zum Markenzeichen von Judith Zaugg geworden. Einziges Problem: Die schönen alten Modelle sind immer schwieriger aufzutreiben. "Jahrelang belieferte mich ein Occasionshändler mit sehr schönen, verschieden grossen Röntgenbildbetrachtern. Nun ist er aber aus diesem Geschäft ausgestiegen. Darum habe ich selber Leuchtkästen bauen lassen, aber der Charme der Röntgenbildbetrachter fehlt mir bei ihnen.» 

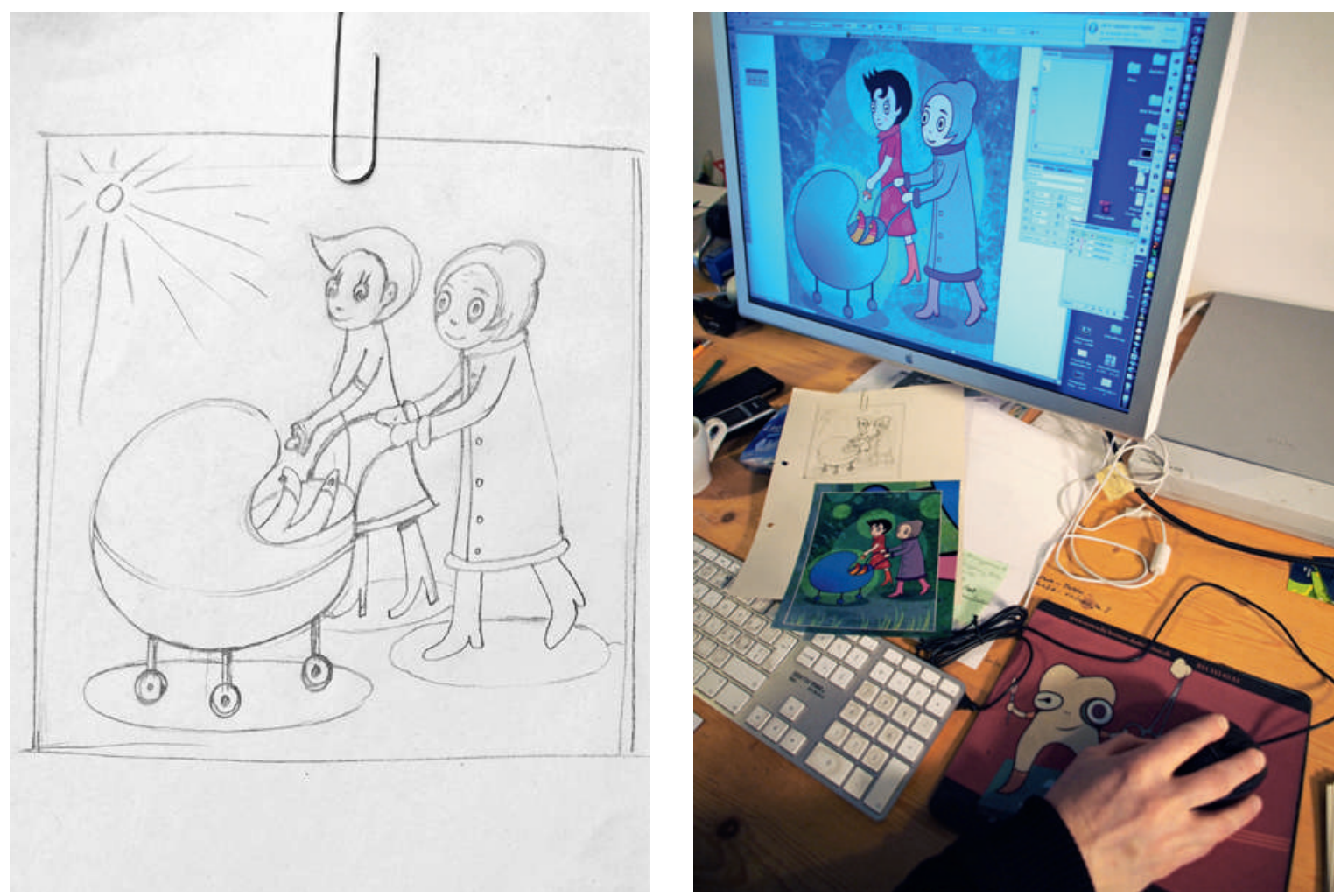

Der kreative Prozess beginnt mit einer Bleistift-Skizze. Gezeichnet - bzw. grafisch konstruiert - wird am Computer.

\section{Wunderbare Renaissance}

Von Charme kann bei diesen Kästen im alltäglichen Einsatz ja nicht die Rede sein. Vielmehr sind sie in der Arztpraxis oft die Vermittler von schlechten Nachrichten; die Orte, wo Leiden sichtbar wird. Ganz anders nach ihrer Wiedergeburt: Da zeigen sie Dinge, die glücklich machen. Hier erlebt medizinisches Alteisen also eine wunderbare Renaissance.

Judith Zauggs Figuren haben - im guten Sinn! - etwas Kindliches. Bisweilen sind es tierische Wesen mit menschlichen Zügen, immer sind sie sehr farbig, oft lassen sie an Comic-Figuren denken und manchmal ein wenig an die Sechzigerjahre. "Ich bin weniger eine Geschichtenerzählerin», erzählt Zaugg, «als vielmehr eine Schöpferin von Stimmungen. Ein Titel kann dafür reichen, «Lunapark〉 zum Beispiel. Auch für meine Ausstellungen gebe ich mir Themen vor, Aufträge an mich selber: Apéro, Schleckzeug, Ufo, auch immer: Fast immer versuche ich, mein Bauchgefühl umzusetzen.» Wenn es um ein Thema wie Schwangerschaft und Geburt geht, ist dies sicher besonders wertvoll. Und so sind also zwei dieser alten Kästen in einer völlig anderen Aufmachung und einer ganz neuen Funktion in die gynäkologische Arztpraxis zurückgekehrt, wo die leuchtende Idee ihren Ursprung hatte. Dort sind sie jetzt nicht mehr dazu da, allfälligen medizinischen Problemen auf die Spur zu kommen und diese schwarz-weiss abzubilden, sondern nur noch dazu, Licht zu spenden und Freude zu bereiten.

Gibt es in der Welt der Medizin ein besseres Beispiel für eine kunstvolle Art von Recycling?

\section{Gesucht: alte Röntgenkästen}

Wer hat noch einen alten Röntgenkasten? Oder wer weiss, wo solche zu haben wären? Grösse spielt keine Rolle. Sachdienliche Hinweise bitte an: contact[at]judithzaugg.ch Vielen Dank! 